\title{
Avaliação do ambiente da prática profissional da enfermagem em instituições de saúde
}

Evaluation of the professional practice environment of nursing in health institutions Evaluación del ambiente de la práctica profesional de enfermería en instituciones de salud

Renata Cristina Gasparino ${ }^{1}$

Thelen Daiana Mendonça Ferreira ${ }^{1}$

Kamila Mariana Adami de Carvalho ${ }^{1}$

Elke Sandra Alves Rodrigues ${ }^{1}$

Juliana Cristina Abatte Tondo²

Vanessa Abreu da Silva ${ }^{1}$

\section{Descritores}

Ambiente de instituições de saúde; Enfermagem; Hospitais privados; Hospitais públicos; Administração de serviços de saúde

Keywords

Health facility environment; Nursing; Hospitals, private; Hospitals, public; Health services

administration

Descriptores

Ambiente de instituciones de salud; Enfermería Hospitales privados; Hospitales públicos; Administración de los servicios de salud

Submetido 28 de Fevereiro de 2019

Aceito

7 de Maio de 2019

\section{Autor correspondente}

Renata Cristina Gasparino

https://orcid.org/0000-0001-8729-4707

E-mail: grenata@unicamp.br

\section{DOI}

http://dx.doi.org/10.1590/1982-

0194201900061

\section{Resumo}

Objetivo: Avaliar o ambiente hospitalar onde a enfermagem exerce sua prática comparando hospitais públicos e privados e descrever as características que receberam avaliação desfavorável $(\leq 2,5$ pontos) na percepção dos profissionais.

Métodos: Estudo comparativo e transversal realizado em cinco hospitais (dois públicos - A e B e três privados - C, D e E) de um município do interior do estado de São Paulo, com 1773 profissionais de enfermagem. Os instrumentos utilizados foram: ficha para caracterização da amostra e a versão brasileira da Practice Environment Scale. Na análise dos dados, foram utilizadas estatísticas descritivas e inferenciais. Para comparar os hospitais foi utilizado o teste Kruskall Wallis, seguido pelo pós-teste de Dunn e a regressão multinomial.

OS hospitais foi utilizado o teste Kruskall Wallis, seguido pelo pos-teste de Dunn e a regressão multinomial.
Resultados: Na comparação dos hospitais, os hospitais D e E alcançaram médias superiores aos demais e diferenças significantes $(p<0,0001)$ foram obtidas com relação às cinco subescalas do instrumento utilizado. Na regressão multinomial, o hospital D obteve 5,8; 0 E 5,2; 0 C 3,0 e o A 2,7 chances de possuir um ambiente mais favorável, quando comparados ao hospital B. Os itens com nota inferior a 2,5 foram associados, especialmente, a falta de oportunidade de desenvolvimento, reconhecimento, gestão participativa e dimensionamento adequado.

Conclusão: Os hospitais privados apresentaram melhor desempenho quando comparados aos públicos e as características que receberam pior avaliação estavam relacionadas à participação dos enfermeiros nos assuntos hospitalares, fundamentos voltados para a qualidade, suporte dos gestores à equipe e adequação de recursos.

\section{Abstract}

Objective: To evaluate the hospital environment where nursing performs its practice comparing public and private hospitals and describing the characteristics that received unfavorable evaluation ( $\leq 2.5$ points) in the professionals' perception.

Methods: A comparative and cross-sectional study was conducted in five hospitals (two public hospitals - A and B; and three private ones - C $D$ and E) from a city in the countryside of the state of São Paulo, with a total of 1773 nursing professionals. The instruments used were: data sheet for characterization of the sample and the Brazilian version of the Practice Environment Scale. In the data analysis, we used descriptive and inferential statistics. To compare the hospitals, we used the Kruskall Wallis test, followed by the Dunn post-test and the multinomial regression. Results: In the comparison of hospitals, D and E hospitals reached mean above the others and significant differences $(\mathrm{p}<0.0001)$ were obtained in relation to the five subscales of the instrument used. In the multinomial regression, hospital D obtained 5.8; hospital E 5.2; hospital C 3.0 and hospital A 2.7 chances of having a more favorable environment when compared to hospital B. Items with a score lower than 2.5 were associated, especially, lack of opportunity for development, recognition, participatory management and proper sizing.

Conclusion: Private hospitals showed better performance when compared to the public ones, and the characteristics that received the worst evaluation were related to the participation of nurses in hospital affairs based on quality, support of the managers to the team and adequacy of resources.

\section{Resumen}

Objetivo: evaluar el ambiente hospitalario donde la enfermería ejerce su práctica comparando hospitales públicos y privados y describir las características que recibieron evaluación desfavorable ( $\leq 2,5$ puntos) según la percepción de los profesionales.

Métodos: estudio comparativo y transversal realizado en cinco hospitales (dos públicos: A y B; tres privados: C, D y E) de un municipio del interior del estado de São Paulo, con 1.773 profesionales de enfermería. Los instrumentos utilizados fueron: ficha para caracterización de la muestra y la versión brasileña de la Practice Environment Scale. En el análisis de los datos, se utilizaron estadísticas descriptivas e inferenciales. Para comparar los hospitales, se utilizó la prueba Kruskall Wallis, seguida de la prueba posterior de Dunn y la regresión multinomial.

Resultados: en la comparación de los hospitales, los hospitales D y E alcanzaron promedios superiores a los demás y se obtuvieron diferencias significativas $(p<0,0001)$ con relación a las cinco subescalas del instrumento utilizado. En la regresión multinomial, el hospital $D$ obtuvo 5,8 ; el E 5,2; el C 3,0 y el A 2,7 chances de poseer un ambiente más favorable, al compararlos con el hospital B. Los ítems con nota inferior a 2,5 fueron asociados, especialmente, a la falta de oportunidad de crecimiento, reconocimiento, gestión participativa y dimensionamiento adecuado. Conclusión: los hospitales privados presentaron mejor desempeño al compararlos con los públicos y las características que recibieron peor evaluación estaban relacionadas con la participación de los enfermeros en los asuntos hospitalarios, fundamentos orientados a la calidad, apoyo de los gestores al equipo y adecuación de recursos.

\section{Como citar:}

Gasparino RC, Ferreira TD, Carvalho KM, Rodrigues ES, Tondo JC, Silva VA. Avaliação do ambiente da prática profissional da enfermagem em instituições de saúde. Acta Paul Enferm. 2019;32(4):449-55. 


\section{Introdução}

Na década de 1980, nos Estados Unidos, houve uma grande preocupação com a falta de profissionais de enfermagem nas instituiçóes de saúde, evidenciada pela presença de quase 100.000 vagas abertas e mais de $80 \%$ dos hospitais com dimensionamento inadequado, decorrente da inabilidade das organizaçóes em atrair e reter profissionais qualificados. ${ }^{(1)}$

Considerando que a equipe de enfermagem é fundamental para o cuidado dos pacientes, pois é responsável por $95 \%$ da assistência que estes recebem durante sua permanência no hospital, a Academia Americana de Enfermagem iniciou, em 1981, uma força tarefa para examinar as características que facilitavam que a enfermagem desenvolvesse a sua prática. ${ }^{(2)}$

As pesquisas tiveram início nos hospitais que eram reconhecidos pela habilidade em atrair e reter profissionais de enfermagem e oferecer uma assistência qualificada e identificaram atributos relacionados à três categorias: 1) administração com um modelo de gestão participativo, liderança qualificada, estrutura descentralizada, participação da equipe em comissóes e políticas de benefícios; 2) prática profissional voltada para a qualidade e 3) desenvolvimento profissional por meio de capacitação e plano de carreira. Dentre os hospitais avaliados, 41 foram selecionados e receberam a designação de Hospital Magnético (HM), devido a sua capacidade em atrair e reter os profissionais de enfermagem. ${ }^{(2)}$

$\mathrm{Na}$ década de 90, a American Nurses Credentialing Center (ANCC) desenvolveu um programa voluntário de reconhecimento para credenciamento formal dos HM e a partir de entáo, pesquisas vêm sendo conduzidas com o intuito de desenvolver e aprimorar instrumentos para avaliar a presença de características que favorecem a prática profissional da enfermagem, bem como avaliar a relação entre essas características e os resultados com pacientes, profissionais e instituiçóes. ${ }^{(3-8)}$

Segundo o International Council of Nurses é de fundamental importância reconhecer os fatores determinantes dos ambientes favoráveis, porque contribuem para a promoção de um cuidado de excelência, maximizando a saúde e bem-estar dos profissionais e melhorando os resultados para os pacientes e o desempenho organizacional. ${ }^{(9)}$
Partindo desse pressuposto, dentre os instrumentos desenvolvidos para essa finalidade, destaca-se a Practice Environment Scale (PES), pois além de possuir propriedades de medida satisfatórias, ${ }^{(10)}$ essa escala permite classificar os ambientes das instituiçôes em mistos, favoráveis e desfavoráveis ${ }^{(11)}$ e, por isso, pode ser utilizada para comparar cenários, predizer resultados e guiar a avaliação de intervençôes. ${ }^{(12)}$

Considerando que a PES foi recentemente validada para a cultura brasileira ${ }^{(13)} \mathrm{e}$, por isso, ainda são escassos os estudos nacionais disponíveis utilizando esse instrumento e a necessidade em se conhecer e comparar o ambiente das instituiçôes para que estratégias possam ser implementadas em busca da melhoria dos resultados, as seguintes perguntas nortearam a presente pesquisa: existem diferenças entre as características do ambiente dos hospitais públicos e privados? Quais características são consideradas mais desfavoráveis com relação à percepção dos profissionais?

Diante do exposto, os objetivos do presente estudo foram avaliar o ambiente hospitalar onde a enfermagem exerce sua prática comparando hospitais públicos e privados e descrever as características que receberam avaliação desfavorável ( $\leq 2,5$ pontos) na percepção dos profissionais.

\section{Métodos}

Trata-se de um estudo comparativo e transversal realizado em uma cidade do interior do estado de São Paulo. Todos os hospitais do município com mais de 100 leitos foram convidados a participar e dentre as dez instituiçôes elegíveis, apenas cinco autorizaram a realização da pesquisa. Os hospitais A e B são públicos e atendem $100 \%$ dos pacientes provenientes do Sistema Único de Saúde (SUS). Os hospitais C e D atendem pacientes provenientes do SUS, particular e da saúde suplementar e possuem acreditação nível dois pela Organização Nacional de Acreditação (ONA) e o hospital E atende pacientes particulares e da saúde suplementar e possui certificação ONA nível três.

A amostra foi definida pela disponibilidade e aceitação dos enfermeiros, técnicos e auxiliares de enfermagem em participar da pesquisa. Todos que atenderam 
aos critérios de inclusão: pertencer à equipe de enfermagem, prestar assistência direta ao paciente e possuir um período de atuação na unidade igual ou superior a três meses, foram convidados a participar da pesquisa.

Aqueles que concordaram, receberam os seguintes instrumentos: uma ficha para caracterização pessoal e profissional da amostra e a versão brasileira da $\mathrm{PES}^{(13)}$ que tem por objetivo avaliar o ambiente da prática profissional da enfermagem por meio de 24 itens distribuídos em cinco subescalas. ${ }^{(10,13)}$

A subescala Participaçáo dos enfermeiros na discussão dos assuntos hospitalares (cinco itens) demonstra o papel e o valor do enfermeiro no amplo contexto hospitalar; Habilidade, liderança e suporte dos coordenadores/supervisores de enfermagem aos enfermeiros/equipe de enfermagem (cinco itens) enfoca o papel do gerente de enfermagem na instituição, englobando competências chaves que um profissional neste cargo precisa desenvolver. ${ }^{(10,13)}$

A subescala Fundamentos de enfermagem voltados para a qualidade do cuidado (sete itens) enfatiza uma filosofia de enfermagem voltada para altos padróes de qualidade do cuidado; Adequação da equipe e de recursos (quatro itens) descreve a necessidade de uma equipe e suporte de recursos adequados para se prover um cuidado com qualidade e Relações colegiais entre enfermeiros e médicos (três itens) caracteriza as relaçóes de trabalho positivas entre médicos e a equipe de enfermagem. ${ }^{(10,13)}$

A escala de medida utilizada é do tipo Likert com quatro pontos, onde os participantes respondem se concordam que determinada característica está presente em seu trabalho diário por meio da escolha de uma entre quatro opçóes: discordo totalmente (um ponto) a concordo totalmente (quatro pontos), ou seja, quanto maior a pontuação, melhor a percepção do profissional sobre o ambiente no qual atua. $\mathrm{O}$ ponto neutro é representado pela pontuação 2,5 e os escores para as subescalas devem ser obtidos pela média dos escores das respostas dos participantes. ${ }^{(10)}$

As instituiçôes que apresentam pontuaçôes com valores acima de 2,5 em nenhuma ou em apenas uma subescala são classificadas com ambientes desfavoráveis; aquelas que atingem pontuaçôes acima de 2,5 em duas ou três subescalas são consideradas com ambientes mistos e hospitais com valores aci- ma de 2,5 em quatro ou em cinco subescalas, são classificados com ambientes favoráveis a prática profissional da enfermagem. ${ }^{(11)}$

A coleta de dados foi realizada entre os meses de novembro de 2017 a julho de 2018. Os profissionais foram abordados de forma individual e àqueles que atenderam aos critérios de inclusão foram explicados os objetivos da pesquisa. Após a assinatura do Termo de Consentimento Livre e Esclarecido, os participantes receberam os instrumentos de coleta e as pesquisadoras aguardaram o preenchimento dos mesmos.

Os dados foram tabulados no Microsoft Excel for Windows ${ }^{\circledast}$ e analisados pelo Statistical Analysis System $^{\circledast}$ (SAS) versão 9.4 e Statistical Package for the Social Sciences ${ }^{\oplus}$ (SPSS) versão 22. Foram calculadas as frequências absolutas, relativas, medidas de posição e dispersão. Para comparar os hospitais foi utilizado o teste Kruskall Wallis, seguido pelo pós-teste de Dunn e a regressão multinomial.

Previamente à realização da pesquisa, foi obtida autorizaçáo dos responsáveis de cada uma das instituiçóes e parecer favorável dos Comitês de Ética em Pesquisa, sob pareceres números 2.331.210 e 2.378.525.

\section{Resultados}

Participaram do estudo 1773 profissionais (61\% de taxa de resposta), com idade média de 38,2 anos $(d p=9,5)$, tempo na função de 11,5 anos $(d p=8,3)$, tempo na unidade de 6,6 anos $(\mathrm{dp}=6,3)$ e o número médio de pacientes sob a responsabilidade do profissional foi de 6,8 $(\mathrm{dp}=6,7)$, sendo 4,3 $(\mathrm{dp}=6,7)$ para os técnicos e auxiliares de enfermagem e 15,8 pacientes $(\mathrm{dp}=6,7)$ para os enfermeiros. A maioria da amostra era do sexo feminino $(81,3 \%)$, possuía ensino médio completo $(60,0 \%)$ e exercia a função de técnico de enfermagem (69,2\%). Com relação ao setor de trabalho, 43,2\% estavam lotados em unidades de internação de pacientes não críticos. A descrição das características que favorecem a prática profissional da enfermagem, bem como a classificação do ambiente em favorável, misto ou desfavorável e a comparação das instituiçóes foram representadas na tabela 1 .

Considerando a amostra total, as médias e medianas encontradas para as subescalas foram, 
Tabela 1. Descrição das características que favorecem a prática profissional da enfermagem, classificação e comparação do ambiente entre as instituições

\begin{tabular}{|c|c|c|c|c|c|c|c|c|c|c|c|c|c|c|c|c|c|c|c|c|c|}
\hline \multirow{2}{*}{ Subescalas da PES } & \multicolumn{4}{|c|}{ A } & \multicolumn{4}{|c|}{ B } & \multicolumn{4}{|c|}{ C } & \multicolumn{4}{|c|}{ D } & \multicolumn{4}{|c|}{$E$} & \multirow{2}{*}{$p$-value $e^{\S}$} \\
\hline & $n$ & $M^{*}$ & $\mathrm{DP}^{\dagger}$ & $\mathrm{Md}^{\ddagger}$ & $n$ & $\mathrm{M}^{*}$ & $\mathrm{DP}^{\dagger}$ & $M d^{\ddagger}$ & $n$ & $M^{*}$ & $\mathrm{DP}^{\dagger}$ & $\mathrm{Md}^{\ddagger}$ & $\mathrm{n}$ & $\mathrm{M}^{*}$ & $\mathrm{DP}^{\dagger}$ & $\mathrm{Md}^{\ddagger}$ & $\mathrm{n}$ & $M^{*}$ & $\mathrm{DP}^{\dagger}$ & $\mathrm{Md}^{\ddagger}$ & \\
\hline Participaçãa ${ }^{\dagger \dagger}$ & 510 & 2,3 & 0,7 & 2,2 & 314 & 1,9 & 0,7 & 1,6 & 242 & 2,4 & 0,7 & 2,4 & 267 & 2,6 & 0,8 & 2,6 & 390 & 2,7 & 0,7 & 2,6 & $<0,0001$ \\
\hline Fundamentos ${ }^{\ddagger}$ & 497 & 2,6 & 0,6 & 2,6 & 311 & 2,2 & 0,7 & 2,0 & 247 & 2,8 & 0,6 & 2,8 & 264 & 2,9 & 0,6 & 2,9 & 384 & 3,0 & 0,6 & 3,0 & $<0,0001$ \\
\hline 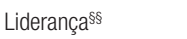 & 511 & 2,6 & 0,7 & 2,6 & 309 & 2,4 & 0,8 & 2,4 & 244 & 2,5 & 0,8 & 2,6 & 266 & 2,9 & 0,7 & 3,0 & 396 & 2,7 & 0,7 & 2,8 & $<0,0001$ \\
\hline Recursos" & 510 & 2,3 & 0,7 & 2,3 & 310 & 1,9 & 0,7 & 1,8 & 245 & 2,4 & 0,7 & 2,5 & 268 & 2,7 & 0,7 & 2,8 & 394 & 2,7 & 0,7 & 2,8 & $<0,0001$ \\
\hline Relações" & 523 & 2,8 & 0,6 & 2,3 & 319 & 2,6 & 0,8 & 2,7 & 251 & 2,6 & 0,7 & 2,7 & 267 & 3,0 & 0,7 & 3,0 & 401 & 2,8 & 0,6 & 3,0 & $<0,0001$ \\
\hline Classificação & \multicolumn{4}{|c|}{ Misto } & \multicolumn{4}{|c|}{ Desfavorável } & \multicolumn{4}{|c|}{ Misto } & \multicolumn{4}{|c|}{ Favorável } & \multicolumn{4}{|c|}{ Favorável } & \\
\hline
\end{tabular}

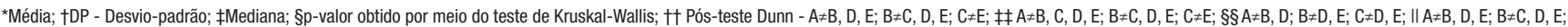
$\mathrm{C} \neq \mathrm{D}, \mathrm{E} ; \uparrow \mathrm{A} \neq \mathrm{B}, \mathrm{D} ; \mathrm{B} \neq \mathrm{D} ; \mathrm{C} \neq \mathrm{D} ; \mathrm{D} \neq \mathrm{E}$

respectivamente: 2,4 e 2,4 para Participação nos assuntos hospitalares; 2,7 e 2,7 para Fundamentos voltados para a qualidade do cuidado; 2,6 e 2,6 para Habilidade da liderança; 2,4 e 2,5 para Adequação dos recursos e 2,8 e 3,0 para Relações entre médicos e enfermeiros. Ainda com relação a comparação dos hospitais, foi realizada uma regressão multinomial para estimar a chance de um hospital apresentar características mais favoráveis com relação ao ambiente onde a enfermagem desenvolve as suas atividades e esses achados foram representados na tabela 2 .

Tabela 2. Chance de um hospital apresentar resultados mais favoráveis com relação às características do ambiente, quando comparado ao hospital B

\begin{tabular}{|c|c|c|c|c|c|}
\hline \multirow{2}{*}{$\begin{array}{l}\text { Variável } \\
\text { dependente }\end{array}$} & \multirow{2}{*}{$\begin{array}{c}\text { Variáveis } \\
\text { independentes }\end{array}$} & \multirow{2}{*}{$\begin{array}{c}\text { Razão de } \\
\text { chances }\end{array}$} & \multicolumn{2}{|c|}{ Intervalo de confiança (95\%) } & \multirow[b]{2}{*}{$p$-value } \\
\hline & & & $\begin{array}{c}\text { Limite } \\
\text { inferior }\end{array}$ & $\begin{array}{l}\text { Limite } \\
\text { superior }\end{array}$ & \\
\hline \multirow{4}{*}{ Ambiente } & A & 2,7 & 2,1 & 3,6 & $<0,0001$ \\
\hline & C & 3,0 & 2,2 & 4,2 & $<0,0001$ \\
\hline & D & 5,8 & 4,2 & 8,1 & $<0,0001$ \\
\hline & $E$ & 5,2 & 3,9 & 7,1 & $<0,0001$ \\
\hline
\end{tabular}

Os itens que alcançaram valores iguais ou inferiores a 2,5, em cada subescala, foram apresentados no quadro 1.
Destaca-se que nenhum item recebeu avaliação inferior a note de corte na subescala Relaçóes entre médicos e enfermeiros.

\section{Discussão}

Na concepção dessa pesquisa, tinha-se por intenção mapear o ambiente da prática profissional da enfermagem nos dez hospitais elegíveis para o estudo, porém apenas cinco autorizaram, o que pode limitar a generalização dos resultados. Além disso, as diferenças encontradas entre os hospitais públicos e privados foram analisadas por meio do financiamento e processo de acreditação, porém, sabe-se que outras variáveis que não foram controladas, podem ter influenciado os resultados.

Os achados deste estudo são importantes para que os gestores conheçam as características do ambiente das instituiçóes nas quais atuam e possam fazer benchmarking, comparando a sua realidade com a de outras instituiçôes. Além disso, essa pesquisa fornece subsídios para a implementação de estratégias que melhor qualifiquem os ambientes, pois a literatura demonstra que em ambientes favoráveis à prática

Quadro 1. Itens que receberam pontuação igual ou inferior a 2,5 nas subescalas da Practice Environment Scale

\begin{tabular}{|l|l|}
\hline Subescala & Itens \\
\hline Participação na discussão dos assuntos & Oportunidade de desenvolvimento na carreira profissional. \\
\cline { 2 - 2 } hospitalares & Oportunidades de aperfeiçoamento. \\
\cline { 2 - 2 } & A administração da instituição ouve e responde às preocupações dos trabalhadores. \\
\cline { 2 - 2 } & O gerente/coordenador/supervisor de enfermagem, da unidade, consulta a equipe sobre os procedimentos e problemas do dia a dia. \\
\hline \multirow{2}{*}{$\begin{array}{l}\text { Fundamentos voltados para a qualidade do } \\
\text { cuidado }\end{array}$} & Programa ativo de garantia da qualidade. \\
\cline { 2 - 2 } & Programa de acompanhamento/tutoria dos profissionais de enfermagem recém-contratados. \\
\hline \multirow{3}{*}{ Habilidade, liderança e suporte dos gestores } & Os gerentes/coordenadores/supervisores, da unidade, utilizam os erros como oportunidades de aprendizagem e não como críticas. \\
\cline { 2 - 2 } & Reconhecimento e elogio por um trabalho bem feito. \\
\hline Adequação da equipe e de recursos & Tempo e oportunidade suficientes para discutir com outros enfermeiros os problemas relacionados aos cuidados do paciente. \\
\cline { 2 - 2 } & Equipe de enfermagem em número suficiente para proporcionar aos pacientes um cuidado de qualidade. \\
\cline { 2 - 2 } & Equipe de enfermagem suficiente para realizar o trabalho. \\
\hline
\end{tabular}


da enfermagem, melhores são os resultados para os pacientes, ${ }^{(14-16)}$ profissionais ${ }^{(17,18)}$ e instituições. ${ }^{(17,19)}$

Na descrição da percepção dos profissionais com relação ao ambiente no qual atuam, foi possível notar que nos hospitais públicos da presente pesquisa (A e B) o ambiente foi classificado, respectivamente, como misto e desfavorável. Em uma pesquisa nacional utilizando a PES em quatro unidades de terapia intensiva de hospitais de ensino, apesar dos autores não terem realizado a classificação do ambiente, foi possível inferir a partir das médias expressas que o ambiente dessas unidades foi desfavorável. Por se tratar de hospitais de ensino, provavelmente públicos, os resultados se assemelham aos encontrados no hospital B, do presente estudo. ${ }^{(20)}$

Com relação aos hospitais privados $(\mathrm{C}, \mathrm{D}$ e E), não foram encontrados estudos utilizando a $\mathrm{PES}$, na cultura brasileira. Entretanto, ao utilizar outro instrumento que avalia as características do ambiente da prática profissional, autores também se depararam com resultados mais favoráveis nos hospitais privados, quando comparados aos públicos. ${ }^{(21)}$

No que se refere às subescalas, a Participação dos enfermeiros nos assuntos hospitalares foi a que obteve avaliação mais desfavorável na percepção dos participantes e ao avaliar países como Estados Unidos, China, Tailândia, Japão, Nova Zelândia, Alemanha ${ }^{(22)}$ e Turquia ${ }^{(23)}$ foi possível perceber que os hospitais pesquisados têm muito a investir na inclusão dos membros da equipe de enfermagem nas decisões políticas, comissóes e comitês, bem como no oferecimento de oportunidades de crescimento profissional e em uma comunicação mais acessível junto aos gestores. ${ }^{(10)}$

$\mathrm{Na}$ Adequação de recursos, segunda subescala com avaliação mais desfavorável, notou-se que apenas a China e a Tailândia alcançaram resultados mais favoráveis, ${ }^{(22,23)}$ o que demonstra que a sensação de sobrecarga de trabalho não se faz presente apenas nos profissionais participantes da presente pesquisa.

Os itens que compóem essa subescala caracterizam, especialmente, o dimensionamento de pessoal e ao analisar o número de pacientes sob responsabilidade do profissional de nível médio notou-se que a média encontrada foi muito próxima a de um estudo realizado em um hospital pediátrico brasileiro. (7) Com relação aos enfermeiros, a média encontrada na presente pesquisa (15,8 pacientes) foi superior ao estudo anteriormente citado (12,4 pacientes), ${ }^{(7)}$ bem como, em outro estudo nacional realizado em terapia intensiva adulto $\left(9,1\right.$ pacientes). ${ }^{(24)}$

Esses achados podem explicar o motivo pelo qual essa subescala náo tenha alcançado valores considerados favoráveis, pois a carga de trabalho, sobretudo dos enfermeiros, foi maior no presente estudo. Além do dimensionamento inadequado da equipe de enfermagem, a falta de serviços de apoio adequados, item também contemplado nessa subescala, contribui para a sobrecarga dos profissionais que, muitas vezes, acabam desenvolvendo atividades que não fazem parte das suas funçóes, em prol dos pacientes.

Na subescala Habilidade e liderança da gestão, os achados demonstraram que países como a China, Tailândia, Nova Zelândia, Reino Unido e Turquia alcançaram resultados mais favoráveis, ${ }^{(22,23)}$ revelando a necessidade de uma revisão da atuação dos gestores junto à equipe. ${ }^{(10)}$

Apesar da subescala Fundamentos voltados para a qualidade do cuidado ter sido a segunda considerada mais favorável, ao comparar o resultado encontrado com os dados de outros nove países, notou-se que os hospitais da presente pesquisa demonstraram resultados mais favoráveis apenas quando comparados a Coréia do Sul ${ }^{(22)}$ e Turquia. ${ }^{(23)} \mathrm{O}$ desenvolvimento da equipe, programas que garantam a qualidade da assistência e planos de cuidados descritos e atualizados para os pacientes ${ }^{(10)}$ são de fundamental importância para o alcance de melhores resultados.

No que se refere à subescala Relaçóes entre médicos e enfermeiros, a mais bem avaliada pelos profissionais que participaram desta pesquisa, foi possível perceber melhor desempenho dos hospitais estudados quando comparados apenas a China, Tailândia e Nova Zelândia. ${ }^{(22)}$ A comunicação entre os profissionais é essencial para se garantir a segurança da assistência prestada aos pacientes. ${ }^{(25)}$

A classificação do ambiente dos hospitais do presente estudo não pode ser comparada à classificação das instituiçóes investigadas na pesquisa que envolveu nove países, ${ }^{(22)}$ pois a amostra de hospitais variou entre 19, no Japão, à 762, nos Estados Unidos, totalizando 1406 instituiçóes. ${ }^{(22)}$ Vale ressaltar que o presente estudo foi realizado em apenas cinco hospitais e considerando a extensão e diversi- 
dade cultural existentes no Brasil, novas investigaçôes, em diferentes regiôes do país, são de extrema importância para que a realidade brasileira possa ser, efetivamente, comparada à internacional.

$\mathrm{Na}$ comparação entre os hospitais, foi possível perceber diferenças significantes com relação à todas as subescalas. Em geral, o hospital privado e os que atendem tanto pacientes provenientes do SUS, quanto particulares e da saúde suplementar, demonstraram melhor desempenho com relação a presença de características favoráveis à prática profissional da enfermagem quando comparados aos públicos, que atendem $100 \%$ dos pacientes provenientes do SUS.

Esses dados também foram confirmados na regressão, na medida em que os hospitais D, E e C (privados) demonstraram maiores chances de possuir um ambiente favorável ao desenvolvimento das atividades da enfermagem quando comparados ao hospital B (público). Apesar do hospital A (público), também ter apresentado maior chance de possuir um ambiente mais favorável do que o $\mathrm{B}$, foi possível notar que os públicos, de um modo geral, apresentaram desempenho mais insatisfatório.

Essas análises permitiram a reflexão de que, talvez, o sistema de financiamento dos hospitais e o processo de acreditação podem, de alguma forma, influenciar o ambiente de trabalho da enfermagem.

De maneira geral, o hospital B, seguido pelo A, foram os que obtiveram pior desempenho com relação à percepção dos profissionais sobre o ambiente no qual atuam. Esses hospitais, por atenderem $100 \%$ dos pacientes provenientes do SUS, enfrentam inúmeros problemas e fragilidades de ordem política, econômica, de gestão e assistência. ${ }^{(26)}$

O subfinanciamento do SUS ${ }^{(26)}$ pode contribuir para que algumas características como oportunidades de aperfeiçoamento, desenvolvimento na carreira profissional, programa ativo de garantia da qualidade, programa de tutoria para recém-contratados e dimensionamento de pessoal, fossem negativamente avaliadas.

O hospital C, além do SUS, complementa seu financiamento atendendo pacientes particulares e provenientes da saúde suplementar (aproximadamente 40\%). Esse hospital é acreditado pleno pela ONA, fato que pode contribuir para a padronização e mapeamento de processos assistenciais, desenvol- vimento da estrutura física, da organização do trabalho, da liderança e da gestão de custos. ${ }^{(27)}$

Os hospitais D e E que alcançaram melhor avaliação possuem, respectiva e aproximadamente $70 \%$ e $100 \%$ do financiamento proveniente de atendimentos particulares e da saúde suplementar, além de contarem com todos os benefícios já descritos advindos do processo de acreditação, pois possuem certificação ONA nível dois e três, respectivamente. Um financiamento mais adequado às necessidades vinculado ao processo de acreditação, podem ter contribuído para que esses hospitais fossem melhor avaliados pelos seus colaboradores, entretanto, outras variáveis, que não foram contempladas na presente pesquisa, influenciam as características do ambiente onde a enfermagem desenvolve suas atividades, pois o hospital $\mathrm{D}$ foi o que apresentou maiores chances de ser um hospital com características favoráveis e ainda não possui ONA nível três e também, possui parte do financiamento proveniente do SUS.

Vale destacar que apesar de alguns itens terem sido avaliados com notas inferiores à de corte estarem direta ou indiretamente ligados a investimentos financeiros, a maioria refere-se a mudanças nos comportamentos dos gestores e na construção de relações respeitosas entre os profissionais. Autores destacam que essas atitudes não adicionam nenhum custo operacional e contribuem para a construção de um ambiente mais favorável à prática da enfermagem. ${ }^{(28)}$

Por fim, considerando que o Brasil é um país muito grande, com diferentes realidades, novos estudos relacionados ao mapeamento das características do ambiente da prática da enfermagem, utilizando o mesmo instrumento e controlando outras variáveis, devem ser realizados para que a realidade nacional possa ser melhor comparada à internacional e para que os gestores possam ter dados que os auxiliem na implementação de novas estratégias com vistas à obtenção de melhores resultados.

\section{Conclusão}

Os hospitais que contam com financiamento proveniente da saúde suplementar e particular apresentaram melhor desempenho quando comparados aos públicos e as características que receberam pior ava- 
liação na percepção dos profissionais estiveram relacionadas à Participação dos enfermeiros nos assuntos hospitalares, Fundamentos voltados para a qualidade do cuidado, Liderança e suporte dos coordenadores à equipe de enfermagem e Adequação de recursos.

\section{Agradecimentos}

Processo no 2016/20030-0, Fundação de Amparo à Pesquisa do Estado de São Paulo (FAPESP). Auxílio à Pesquisa - Regular para Renata Cristina Gasparino.

\section{Colaborações}

Gasparino RC, Ferreira TDM, Carvalho KMA, Rodrigues ESA, Tondo JCA e Silva VA declaram que contribuíram com a concepção do estudo, análise e interpretação dos dados, redação do artigo, revisão crítica relevante do conteúdo intelectual e aprovação da versão final a ser publicada.

\section{Referências}

1. Aiken LH. Nursing priorities for the 1980's: hospitals and nursing homes. Am J Nurs. 1981;81(2):324-30.

2. McClure ML, Poulin MA, Sovie MD, Wandelt MA. Magnet hospitals: attraction and retention of professional nurses (the original study). In: McClure ML, Hinshaw AS, editors. Magnet hospitals revisited: attraction and retention of professional nurses. Washington: American Nurses Publishing; 2002. p. 1-24.

3. Kanai-Pak M, Aiken LH, Sloane DM, Poghosyan L. Poor work environments and nurse inexperience are associated with burnout, job dissatisfaction and quality deficits in Japanese hospitals. J Clin Nurs. 2008;17(24):3324-9.

4. Aiken LH, Cimiotti JP, Sloane DM, Smith HL, Flynn L, Neff DF. Effects of nurse staffing and nurse education on patient deaths in hospitals with different nurse work environments. Med Care. 2011;49(12):1047-53.

5. Kutney-Lee A, Wu ES, Sloane DM, Aiken LH. Changes in hospital nurse work environments and nurse job outcomes: an analysis of panel data. Int J Nurs Stud. 2013 Feb;50(2):195-201.

6. Gasparino RC, Guirardello EB. Professional practice environment and burnout among nurses. Rev Rene. 2015;16(1):90-6.

7. AlvesDF, GuirardelloEB. Nursing workenvironment, patient safety and quality of care in pediatric hospital. Rev Gaúcha Enferm. 2016;37(2):e58817.

8. Dorigan GH, Guirardello EB. Effect of the practice environment of nurses on job outcomes and safety climate. Rev Lat Am Enfermagem. 2018;26:e3056.
9. Oulton JA. The global nursing shortage: an overview of issues and actions. Policy Polit Nurs Pract. 2006;7(3 Suppl):34S-9S.

10. Lake ET. Development of the practice environment scale of the Nursing Work Index. Res Nurs Health. 2002;25(3):176-88.

11. Lake ET, Friese CR. Variations in nursing practice environments: relation to staffing and hospital characteristics. Nurs Res. 2006;55(1):1-9.

12. Lake ET. The nursing practice environment: measurement and evidence. Med Care Res Rev. 2007;64(2 Suppl):104S-22S.

13. Gasparino RC, Guirardello EB. Validation of the Practice Environment Scale to the Brazilian culture. J Nurs Manag. 2017;25(5):375-83.

14. Olds DM, Aiken LH, Cimiotti JP, Lake ET. Association of nurse work environment and safety climate on patient mortality: A cross-sectional study. Int J Nurs Stud. 2017;74:155-61.

15. Aiken LH, Sloane DM, Ball J, Bruyneel L, Rafferty AM, Griffiths P. Patient satisfaction with hospital care and nurses in England: an observational study. BMJ Open. 2018;8(1):e019189.

16. McHugh MD, Rochman MF, Sloane DM, Berg RA, Mancini ME, Nadkarni VM, et al.; American Heart Association's Get with The GuidelinesResuscitation Investigators. Better nurse staffing and nurse work environments associated with increased survival of in-hospital cardiac arrest patients. Med Care. 2016;54(1):74-80.

17. Nantsupawat A, Kunaviktikul W, Nantsupawat $R$, Wichaikhum $O A$, Thienthong H, Poghosyan L. Effects of nurse work environment on job dissatisfaction, burnout, intention to leave. Int Nurs Rev. 2017;64(1):91-8.

18. Nogueira LS, Sousa RM, Guedes ES, Santos MA, Turrini RN, Cruz DA. Burnout and nursing work environment in public health institutions. Rev Bras Enferm. 2018;71(2):336-42.

19. Park SH, Gass S, Boyle DK. Comparison of reasons for nurse turnover in magnet and non-magnet hospitals. J Nurs Adm. 2016;46(5):284-90.

20. Azevedo Filho FM, Rodrigues MC, Cimiotti JP. Nursing practice environment in intensive care units. Acta Paul Enferm. 2018;31(2):217-23.

21. Pires BS, Oliveira LZ, Siqueira CL, Feldman LB, Oliveira RA, Gasparino RC. Nurse work environment: comparison between private and public hospitals. einstein (São Paulo). 2018;16(4):eA04322.

22. Aiken LH, Sloane DM, Clarke S, Poghosyan L, Cho E, You L, et al. Importance of work environments on hospital outcomes in nine countries. Int J Qual Health Care. 2011;23(4):357-64.

23. Topçu I, Türkmen E, Badır A, Göktepe N, Miral M, Albayrak S, et al. Relationship between nurses' practice environments and nursing outcomes in Turkey. Int Nurs Rev. 2016;63(2):242-9.

24. Panunto MR, Guirardello EB. Professional nursing practice: environment and emotional exhaustion among intensive care nurses. Rev Lat Am Enfermagem. 2013;21(3):765-72.

25. Nogueira JW, Rodrigues MC. Effective communication in teamwork in health: a challenge for patient safety. Cogitare Enferm. 2015;20(3):630-4.

26. Reis AA, Sóter AP, Furtado LA, Pereira SS. Tudo a temer: financiamento, relação público e privado e o futuro do SUS. Saúde Debate. 2016;40(N Espec):122-35.

27. Oliveira JL, Gabriel CS, Fertonani HP, Matsuda LM. Management changes resulting from hospital accreditation. Rev LatAm Enfermagem. 2017;25(0):e2851.

28. Aiken LH. Superior outcomes for magnet hospitals: the evidence base. In: McClure ML, Hinshaw AS, editors. Magnet hospitals revisited: attraction and retention of professional nurses. Washington: American Nurses Publishing; 2002. p. 61-81. 